PROCEEDINGS OF THE

AMERICAN MATHEMATICAL SOCIETY

Volume 137, Number 6, June 2009, Pages 1899-1906

S 0002-9939(09)09663-4

Article electronically published on January 21, 2009

\title{
CYCLES ON NASH ALGEBRAIC MODELS OF SMOOTH MANIFOLDS
}

\author{
WOJCIECH KUCHARZ
}

(Communicated by Paul Goerss)

\begin{abstract}
A Nash algebraic model of a compact smooth manifold $M$ is a pair $\left(X, X_{0}\right)$ where $X$ is a nonsingular real algebraic set and $X_{0}$ is the union of some connected components of $X$ such that $X_{0}$ is diffeomorphic to $M$. We study the homology classes on $X_{0}$ represented by algebraic subsets of $X$ contained in $X_{0}$ for various Nash algebraic models $\left(X, X_{0}\right)$ of $M$.
\end{abstract}

\section{INTRODUCTION}

In 1973 A. Tognoli [25, improving upon an earlier result of J. Nash 20], demonstrated that every compact smooth (of class $\mathcal{C}^{\infty}$ ) manifold $M$ is diffeomorphic to a nonsingular real algebraic set (in $\mathbb{R}^{n}$ for some $n$ ), henceforth called an algebraic model of $M$. Since then a lot of effort has been devoted to the construction of algebraic models with certain additional desirable properties; see 1, 2, 3, 4, 5, 6, 7, 8, 9, 18. In the recent papers [13, 14, 15, 19, a relaxed concept of algebraic model is used, which is closer in spirit to Nash's original paper 20]. By a Nash algebraic model of $M$, we mean a pair $\left(X, X_{0}\right)$, where $X$ is a compact nonsingular real algebraic set, $X_{0}$ is the union of some connected components of $X$, and $X_{0}$ is diffeomorphic to $M$. Note that $X \backslash X_{0}$ is a smooth manifold, being either empty or a union of connected components of $X$. We say that the Nash algebraic model $\left(X, X_{0}\right)$ of $M$ is nice if $X \backslash X_{0}$ is diffeomorphic to $M$, in which case the topology of $X$ is determined by $M$. All Nash algebraic models constructed in this paper happen to be nice.

In order to state our results we need some preparation. A homology class in $H_{k}(X, \mathbb{Z} / 2)$, where $X$ is a compact nonsingular real algebraic set, is said to be algebraic if it can be represented by a $k$-dimensional algebraic subset of $X$. The set $H_{k}^{\text {alg }}(X, \mathbb{Z} / 2)$ of all algebraic homology classes in $H_{k}(X, \mathbb{Z} / 2)$ is a subgroup. Early papers dealing with algebraic homology classes provided examples of $X$ with $H_{k}^{\text {alg }}(X, \mathbb{Z} / 2) \neq H_{k}(X, \mathbb{Z} / 2)$ for some $k$; see 11, 5, 6, 16, 21, 22. The groups $H_{k}^{\text {alg }}(X, \mathbb{Z} / 2)$ play an important role in real algebraic geometry. Their properties and applications are surveyed in 10. If $X_{0}$ is the union of some connected components of $X$, we denote by $H_{k}^{\text {alg }}\left(X_{0}, \mathbb{Z} / 2\right)$ the subgroup of $H_{k}(X, \mathbb{Z} / 2)$ consisting of

Received by the editors April 24, 2008, and, in revised form, June 7, 2008.

2000 Mathematics Subject Classification. Primary 14P05, 14P25, 57R19.

Key words and phrases. Real algebraic sets, algebraic homology classes, algebraic models, Nash algebraic models. 
the homology classes represented by $k$-dimensional algebraic subsets of $X$ contained in $X_{0}$.

The behavior of the groups $H_{k}^{\mathrm{alg}}(X, \mathbb{Z} / 2)$ (or their counterparts in cohomology) as $X$ runs through the class of all algebraic models of a given compact smooth manifold $M$ is investigated in 7, , 8, 9, 18.

In the present paper, we study the groups $H_{k}^{\text {alg }}\left(X_{0}, \mathbb{Z} / 2\right)$ as $\left(X, X_{0}\right)$ runs through the class of all Nash algebraic models of $M$.

Given homology classes $\alpha$ in $H_{k}(M, \mathbb{Z} / 2)$ and $\beta$ in $H_{m-k}(M, \mathbb{Z} / 2), m=\operatorname{dim} M$, we denote by $\alpha \bullet \beta$ their intersection number in $\mathbb{Z} / 2$. Recall that

$$
\alpha \bullet \beta=\left\langle D_{M}^{-1}(\alpha) \cup D_{M}^{-1}(\beta),[M]\right\rangle,
$$

where $[M]$ is the fundamental class of $M$ in $H_{m}(M, \mathbb{Z} / 2)$ and

$$
D_{M}: H^{*}(M, \mathbb{Z} / 2) \rightarrow H_{*}(M, \mathbb{Z} / 2), \quad D_{M}(v)=v \cap[M]
$$

is the Poincaré duality isomorphism.

Theorem 1.1. Let $M$ be a compact connected smooth manifold and let $G$ be a subgroup of $H_{k}(M, \mathbb{Z} / 2)$, where $1 \leq k \leq m / 2, m=\operatorname{dim} M \geq 3$. Assume that the subgroup

$$
\bar{G}=\left\{\beta \in H_{m-k}(M, \mathbb{Z} / 2) \mid \alpha \bullet \beta=0 \text { for all } \alpha \in G\right\}
$$

of $H_{m-k}(M, \mathbb{Z} / 2)$ is generated by the homology classes represented by some $(m-k)$ dimensional smooth submanifolds of $M$ with trivial normal bundle. Then there exist a nice Nash algebraic model $\left(X, X_{0}\right)$ of $M$ and a smooth diffeomorphism $\varphi: M \rightarrow$ $X_{0}$ such that $\varphi_{*}(G)=H_{k}^{\text {alg }}\left(X_{0}, \mathbb{Z} / 2\right)$.

Theorem 1.1 is nontrivial even when $G=0$; however, it is then a special case of our next result.

Theorem 1.2. Any compact smooth manifold $M$ of dimension $m$ has a nice Nash algebraic model $\left(X, X_{0}\right)$ such that $H_{k}^{\text {alg }}\left(X_{0}, \mathbb{Z} / 2\right)=0$ for each $k \geq 1$, for which the group $H_{m-k}(M, \mathbb{Z} / 2)$ is generated by the homology classes represented by $(m-k)$ dimensional smooth submanifolds of $M$ with trivial normal bundle.

According to Theorem 1.2, the $m$-torus $\mathbb{R}^{m} / \mathbb{Z}^{m}$ has a nice Nash algebraic model $\left(X, X_{0}\right)$ such that $H_{k}^{\text {alg }}\left(X_{0}, \mathbb{Z} / 2\right)=0$ for all $k \geq 1$. It is not known whether for odd $m$ greater than 2 there is an algebraic model $Y$ of $\mathbb{R}^{m} / \mathbb{Z}^{m}$ with $H_{1}^{\text {alg }}(Y, \mathbb{Z} / 2)=0$.

Theorems 1.1 and 1.2 are proved in the next section.

\section{Proofs}

Let $B^{k}$ be a nonsingular irreducible real algebraic set with precisely two connected components $B_{0}^{k}$ and $B_{1}^{k}$, each diffeomorphic to the unit $k$-sphere, $k \geq 1$. For example, one can take

$$
B^{k}=\left\{\left(x_{0}, \ldots, x_{k}\right) \in \mathbb{R}^{k+1} \mid x_{0}^{4}-4 x_{0}^{2}+1+x_{1}^{2}+\cdots+x_{k}^{2}=0\right\} .
$$

Let

$$
B^{k}(d)=B^{k} \times \cdots \times B^{k} \text { and } B_{0}^{k}(d)=B_{0}^{k} \times \cdots \times B_{0}^{k}
$$

be the $d$-fold products, $d \geq 1$. 
Lemma 2.1. Let $X$ be a compact nonsingular real algebraic set and let $X_{0}$ be the union of some connected components of $X$. If $g: X \rightarrow B^{k}(d)$ is a regular map such that $g\left(X_{0}\right) \subseteq B_{0}^{k}(d)$, then

$$
\left(g \mid X_{0}\right)_{*}(\beta)=0 \text { in } H_{k}\left(B^{k}(d), \mathbb{Z} / 2\right)
$$

for every homology class $\beta$ in $H_{k}^{\text {alg }}\left(X_{0}, \mathbb{Z} / 2\right)$.

Proof. In 17, 18, for every compact nonsingular real algebraic set $Y$ a certain subgroup $\operatorname{Alg}^{k}(Y)$ of $H^{k}(Y, \mathbb{Z} / 2)$ is defined. If $w$ is in $\operatorname{Alg}^{k}(Y)$ and $\gamma$ is in $H_{k}^{\text {alg }}(Y, \mathbb{Z} / 2)$, then

$$
\langle w, \gamma\rangle=0 .
$$

Indeed, $(i)$ is equivalent to [18, Theorem 4.4] (and to [17, Theorem 2.1]), since

$$
\langle w, \gamma\rangle=\left\langle w, D_{Y}^{-1}(\gamma) \cap[Y]\right\rangle=\left\langle w \cup D_{Y}^{-1}(\gamma),[Y]\right\rangle .
$$

Moreover, if $\delta: B_{0}^{k}(d) \hookrightarrow B^{k}(d)$ is the inclusion map, then

$$
H^{k}\left(B_{0}^{k}(d), \mathbb{Z} / 2\right)=\delta^{*}\left(\operatorname{Alg}^{k}\left(B^{k}(d)\right)\right)
$$

cf. [18, Example 4.5]. Both $(i)$ and (ii) will be crucial.

Let $\beta$ be a homology class in $H_{k}^{\text {alg }}\left(X_{0}, \mathbb{Z} / 2\right)$. Then $i_{*}(\beta)$ is in $H_{k}^{\text {alg }}(X, \mathbb{Z} / 2)$, where $i: X_{0} \hookrightarrow X$ is the inclusion map. Hence $g_{*}\left(i_{*}(\beta)\right)$ is in $H_{k}^{\text {alg }}\left(B^{k}(d), \mathbb{Z} / 2\right)$, the map $g$ being regular; see [6, 11]. Denoting by $\bar{g}: X_{0} \rightarrow B_{0}^{k}(d)$ the restriction of $g$, we have $\delta \circ \bar{g}=g \circ i$, and hence

$$
\left\langle\delta^{*}(v), \bar{g}_{*}(\beta)\right\rangle=\left\langle v, \delta_{*}\left(\bar{g}_{*}(\beta)\right)\right\rangle=\left\langle v, g_{*}\left(i_{*}(\beta)\right)\right\rangle=0
$$

for every $v$ in $\operatorname{Alg}^{k}\left(B^{k}(d)\right)$, where the last equality follows from $(i)$. Making use of (ii) and $\left(\right.$ iii), we get $\bar{g}_{*}(\beta)=0$. Since $g \mid X_{0}=\delta \circ g$, we obtain $(g \mid X)_{*}(\beta)=0$, as required.

Let $M$ be a compact smooth manifold. Given an $n$-dimensional smooth submanifold $N$ of $M$ (always assumed to be a closed subset of $M$ ), we set

$$
[N]_{M}:=i_{*}([N])
$$

where $i: N \hookrightarrow M$ is the inclusion map. In other words, $[N]_{M}$ is the homology class in $H_{n}(M, \mathbb{Z} / 2)$ represented by $N$.

A cohomology class $v$ in $H^{k}(M, \mathbb{Z} / 2), k \geq 1$, is said to be spherical provided $v=f^{*}(c)$, where $f: M \rightarrow S^{k}$ is a continuous (or equivalently smooth) map from $M$ into the unit $k$-sphere $S^{k}$ and $c$ is the unique generator of $H^{k}\left(S^{k}, \mathbb{Z} / 2\right) \cong \mathbb{Z} / 2$. It is well known that $v$ is spherical if and only if the homology class $D_{M}(v)$ that is Poincaré dual to $v$ can be represented by a smooth submanifold of $M$ with trivial normal bundle; see [24, Théorème II.2].

Proof of Theorem 1.1. Since $k \leq m / 2$, according to [24, Théorème II.26] there exist $k$-dimensional smooth submanifolds $K_{1}, \ldots, K_{\ell}$ of $M$ such that

$$
\left[K_{1}\right]_{M}, \ldots,\left[K_{\ell}\right]_{M} \text { generate } G \text {. }
$$

We may assume that each submanifold $K_{i}, 1 \leq i \leq \ell$, is connected and, for $k \geq 2$, nonorientable.

To justify this claim, we argue as follows. Since $M$ is connected and $k \geq 1, K_{i}$ can be replaced by a connected smooth submanifold of $M$ obtained by joining the connected components of $K_{i}$ with $k$-dimensional tubes. If $k \geq 2$, then $m-k \geq 2$, 
and hence there is a $k$-dimensional connected nonorientable smooth submanifold $P$ of $M$ which is contained in an open subset of $M$ diffeomorphic to $\mathbb{R}^{m}$. It follows that for $k \geq 2, K_{i}$ can be replaced by a nonorientable smooth submanifold of $M$ obtained by joining $K_{i}$ and $P$ with a $k$-dimensional tube. The claim is proved.

By transversality, the submanifolds $K_{1}, \ldots, K_{\ell}$ can be chosen in general position. Let

$\left(a_{2}\right) \quad \Gamma:=\left\{v \in H^{k}(M, \mathbb{Z} / 2) \mid\langle v, \alpha\rangle=0\right.$ for all $\left.\alpha \in G\right\}$.

Since for all $v$ in $H^{k}(M, \mathbb{Z} / 2)$ and $\alpha$ in $H_{k}(M, \mathbb{Z} / 2)$,

$$
\begin{aligned}
\langle v, \alpha\rangle & =\left\langle v, D_{M}^{-1}(\alpha) \cap[M]\right\rangle \\
& =\left\langle v \cup D_{M}^{-1}(\alpha),[M]\right\rangle \\
& =\left\langle D_{M}^{-1}(\alpha) \cup v,[M]\right\rangle \\
& =\alpha \bullet D_{M}(v),
\end{aligned}
$$

it follows that $D_{M}(\Gamma)=\bar{G}$. Therefore the assumption about $\bar{G}$ implies that $\Gamma$ is generated by spherical cohomology classes. Hence, with notation as in Lemma 2.1, there exist a positive integer $d$ and a smooth map

$$
f=\left(f_{1}, \ldots, f_{d}\right): M \rightarrow B^{k}(d)=B^{k} \times \cdots \times B^{k}
$$

satisfying

$$
f(M) \subseteq B_{0}^{k}(d) \text { and } \Gamma=f^{*}\left(H^{k}\left(B^{k}(d), \mathbb{Z} / 2\right)\right)
$$

By $\left(a_{2}\right)$,

$$
G=\left\{\alpha \in H_{k}(M, \mathbb{Z} / 2) \mid\langle v, \alpha\rangle=0 \text { for all } v \in \Gamma\right\} .
$$

Since $\left\langle f^{*}(w), \alpha\right\rangle=\left\langle w, f_{*}(\alpha)\right\rangle$, we have that for every $w$ in $H^{k}\left(B^{k}(d), \mathbb{Z} / 2\right)$ and every $\alpha$ in $H_{k}(M, \mathbb{Z} / 2)$, the equality in $\left(a_{3}\right)$ gives

$$
G=\left\{\alpha \in H_{k}(M, \mathbb{Z} / 2) \mid f_{*}(\alpha)=0\right\} .
$$

Let $M^{\prime}$ be a smooth manifold, $G^{\prime}$ be a subgroup of $H_{k}\left(M^{\prime}, \mathbb{Z} / 2\right), K_{1}^{\prime}, \ldots, K_{\ell}^{\prime}$ be smooth submanifolds of $M^{\prime}$, and $f^{\prime}: M^{\prime} \rightarrow B^{k}(d)$ be a smooth map. We say that $\left(M^{\prime}, G^{\prime} ; K_{1}^{\prime}, \ldots, K_{\ell}^{\prime}, f^{\prime}\right)$ is a modification of $\left(M, G ; K_{1}, \ldots, K_{\ell}, f\right)$ if there exists a smooth diffeomorphism $h: M \rightarrow M^{\prime}$ such that $G^{\prime}=h_{*}(G), K_{i}^{\prime}=h\left(K_{i}\right)$ for $1 \leq i \leq \ell$, and $f^{\prime}$ is homotopic to $f \circ h^{-1}$. Clearly, in the proof we may replace $\left(M, G ; K_{1}, \ldots, K_{\ell}, f\right)$ by a modification.

Assertion. Replacing $\left(M, G ; K_{1}, \ldots, K_{\ell}, f\right)$ by a suitable modification if necessary, we may assume that $M$ is a nonsingular real algebraic set, $K_{1}, \ldots, K_{\ell}$ are nonsingular algebraic subsets of $M$, and $f \mid K: K \rightarrow B^{k}(d)$ is a regular map, where

$$
K:=K_{1} \cup \cdots \cup K_{\ell} .
$$

In the proof of the Assertion, we will consider separately the two cases $k \geq 2$ and $k=1$.

Case 1. Suppose $k \geq 2$. Since $K_{1}, \ldots, K_{\ell}$ are in general position in $M$, by [3, Theorem 2.8.9] or [4, Theorem 4] we may assume that $M$ is a nonsingular algebraic subset of $\mathbb{R}^{n}$ and $K_{1}, \ldots, K_{\ell}$ are nonsingular algebraic subsets of $M$.

We will now prove that the map $f_{j 0} \mid K: K \rightarrow B_{0}^{k}$ is null homotopic, where $f_{j 0}: M \rightarrow B_{0}^{k}$ is defined by $f_{j 0}(x)=f_{j}(x)$ for all $x$ in $M$. Recall that $B_{0}^{k}$ is diffeomorphic to $S^{k}$. By Hopf's classification theorem, $f_{j 0} \mid K$ is null homotopic if and only if $\left(f_{j 0} \mid K\right)^{*}(b)=0$ in $H^{k}(K, \mathbb{Z})$, where $b$ is a generator of $H^{k}\left(B_{0}^{k}, \mathbb{Z}\right) \cong \mathbb{Z}$. 
By the Mayer-Vietoris exact sequence, the equality $\left(f_{j 0} \mid K\right)^{*}(b)=0$ in $H^{k}(K, \mathbb{Z})$ is equivalent to $\left(f_{j 0} \mid K_{i}\right)^{*}(b)=0$ in $H^{k}\left(K_{i}, \mathbb{Z}\right)$ for $1 \leq i \leq \ell$. Since $K_{i}$ is a connected and nonorientable manifold, $\left(f_{j 0} \mid K_{i}\right)^{*}(b)=0$ in $H^{k}\left(K_{i}, \mathbb{Z}\right)$ if and only if $\left(f_{j 0} \mid K_{i}\right)_{*}\left(\left[K_{i}\right]\right)=0$ in $H_{k}\left(B_{0}^{k}, \mathbb{Z} / 2\right)$. We have

$$
\left(f_{j 0} \mid K_{i}\right)_{*}\left(\left[K_{i}\right]\right)=\left(f_{j 0}\right)_{*}\left(\left[K_{i}\right]_{M}\right)=0,
$$

where the last equality follows from $\left(a_{1}\right)$ and $\left(a_{4}\right)$. Hence the map $f \mid K: K \rightarrow B^{k}(d)$ is null homotopic.

In order to complete the proof of the Assertion for $k \geq 2$, it suffices to show that $f: M \rightarrow B^{k}(d)$ is homotopic to a smooth map which is constant on $K$. This can be done as follows. Choose a compact subset $A$ of $M$ such that $(M, A)$ is a polyhedral pair, $K$ is contained in the interior of $A$, and $K$ is a deformation retract of $A$. Then $f \mid A: A \rightarrow B^{k}(d)$ is null homotopic and, by the homotopy extension theorem [23, p. 118, Corollary 5], there exists a continuous map $f^{\prime}: M \rightarrow B^{k}(d)$ which is homotopic to $f$ and such that $f^{\prime} \mid A: A \rightarrow B^{k}(d)$ is a constant map. Thus one can find a smooth map $f^{\prime \prime}: M \rightarrow B^{k}(d)$ that is homotopic to $f^{\prime}$ and equal to $f^{\prime}$ on $K$. Since $f^{\prime \prime}$ is homotopic to $f$ and is constant on $K$, the proof of the Assertion for $k \geq 2$ is complete.

Case 2. Suppose now that $k=1$. We have

$$
\left(f \mid K_{i}\right)_{*}\left(\left[K_{i}\right]\right)=f_{*}\left(\left[K_{i}\right]_{M}\right)=0,
$$

where the last equality follows from $\left(a_{1}\right)$ and $\left(a_{4}\right)$. Consequently, the map $f \mid K_{i}$ : $K_{i} \rightarrow B^{k}(d)$ represents 0 in the unoriented bordism group $\mathcal{N}_{*}\left(B^{k}(d)\right.$ ) (this is elementary, but see [12, (17.3)] if desired). By [3, Theorem 2.8.4], there exist a nonsingular real algebraic set $K_{i}^{\prime}$, a regular map $r_{i}: K_{i}^{\prime} \rightarrow B^{k}(d)$, and a smooth diffeomorphism $h_{i}: K_{i} \rightarrow K_{i}^{\prime}$ such that $r_{i}$ is homotopic to $\left(f \mid K_{i}\right) \circ h_{i}^{-1}$. We may assume that $K_{1}^{\prime}, \ldots, K_{\ell}^{\prime}$ are pairwise disjoint nonsingular algebraic subsets of $\mathbb{R}^{n}$, $n \geq 2 m+1$. Hence one can find a smooth embedding $e: M \rightarrow \mathbb{R}^{n}$ such that $e(x)=h_{i}(x)$ for all $x$ in $K_{i}, 1 \leq i \leq \ell$ (note that $K_{1}, \ldots, K_{\ell}$ are pairwise disjoint, as they are in general position in $M, \operatorname{dim} M=m \geq 3)$. Let $M^{\prime}:=e(M)$ and let $\bar{e}: M \rightarrow M^{\prime}$ be a smooth diffeomorphism defined by $\bar{e}(x)=e(x)$ for all $x$ in $M$. Thus $M^{\prime}$ is a smooth submanifold of $\mathbb{R}^{n}$ containing $K_{1}^{\prime} \cup \cdots \cup K_{\ell}^{\prime}$, and the map $\left(f \circ \bar{e}^{-1}\right) \mid K_{i}^{\prime}$ is homotopic to $r_{i}$ for $1 \leq i \leq \ell$. By the homotopy extension theorem (see the argument in Case 1 above), there exists a smooth map $r: M^{\prime} \rightarrow B^{k}(d)$ homotopic to $f \circ \bar{e}^{-1}$ and with $r \mid K_{i}^{\prime}=r_{i}$ for $1 \leq i \leq \ell$.

Therefore, without loss of generality, we may assume that $M$ is a smooth submanifold of $\mathbb{R}^{n}$, the original $K_{1}, \ldots, K_{\ell}$ are nonsingular algebraic subsets of $\mathbb{R}^{n}$, and $f \mid K: K \rightarrow B^{k}(d)$ is a regular map. Since $\operatorname{dim} K=1$, every topological real vector bundle on $K$ is isomorphic to an algebraic (strongly algebraic, in the terminology used in [5]) vector bundle; see [7, Theorem 12.5.1]. By [5, Theorem 3.2], there exist a nonsingular algebraic subset $M^{\prime \prime}$ of $\mathbb{R}^{n}$ and a smooth diffeomorphism $\theta: M \rightarrow M^{\prime \prime}$ such that $K \subseteq M^{\prime \prime}$ and $\theta(x)=x$ for all $x$ in $K$. This completes the proof of the Assertion for $k=1$.

Henceforth we assume that $M, K_{1}, \ldots, K_{\ell}, f$ are as in the Assertion. In addition, we assume that $M$ is a nonsingular algebraic subset of $\mathbb{R}^{n}$. For any nonnegative integer $p$, we regard $\mathbb{R}^{n}$ as a subset of $\mathbb{R}^{n+p}$, identifying $\mathbb{R}^{n}$ with $\mathbb{R}^{n} \times\{0\} \subseteq \mathbb{R}^{n} \times \mathbb{R}^{p}=\mathbb{R}^{n+p}$. Let $t: \mathbb{R}^{n} \rightarrow \mathbb{R}^{n}$ be a translation such that $M \cap t(M)=\emptyset$, and let $\bar{M}:=M \cup t(M)$. Thus $\bar{M}$ is the disjoint union of two copies 
of $M$. Define

$$
\bar{f}: \bar{M} \rightarrow B^{k}(d)
$$

by $\bar{f}(x)=\bar{f}(t(x))=f(x)$ for all $x$ in $M$. Clearly, the map $\bar{f}: \bar{M} \rightarrow B^{k}(d)$ represents 0 in the unoriented bordism group $\mathcal{N}_{*}\left(B^{k}(d)\right)$. Moreover, $\bar{f}|K=f| K$ is a regular map. By [3, Theorem 2.8.4], there exist a nonnegative integer $p$, a nonsingular algebraic subset $X$ of $\mathbb{R}^{n+p}$, a regular map $g: X \rightarrow B^{k}(d)$, and a smooth diffeomorphism $\psi: \bar{M} \rightarrow X$ such that $K \subseteq X, \psi(x)=x$ for all $x$ in $K$, and $g$ is homotopic to $\bar{f} \circ \psi^{-1}$. Set $X_{0}:=\psi(M)$ and let $\varphi: M \rightarrow X_{0}$ be the restriction of $\psi$. Then $\left(X, X_{0}\right)$ is a Nash algebraic model of $M$, and $\varphi$ is a smooth diffeomorphism.

It remains to prove that $\varphi_{*}(G)=H_{k}^{\text {alg }}(X, \mathbb{Z} / 2)$. This can be done as follows. By construction, $\varphi_{*}\left(\left[K_{i}\right]_{M}\right)=\left[K_{i}\right]_{X_{0}}$ for $1 \leq i \leq \ell$, and hence in view of $\left(a_{1}\right)$,

$$
\varphi_{*}(G) \subseteq H_{k}^{\mathrm{alg}}\left(X_{0}, \mathbb{Z} / 2\right) .
$$

Note that $g \mid X_{0}$ is homotopic to $\left(f \circ \psi^{-1}\right) \mid X_{0}=f \circ \varphi^{-1}$, and hence $\left(a_{4}\right)$ implies

$$
\varphi_{*}(G)=\left\{\beta \in H_{k}\left(X_{0}, \mathbb{Z} / 2\right) \mid\left(g \mid X_{0}\right)_{*}(\beta)=0\right\} .
$$

If $\beta$ is in $H_{k}^{\text {alg }}\left(X_{0}, \mathbb{Z} / 2\right)$, then according to Lemma $2.1,\left(g \mid X_{0}\right)_{*}(\beta)=0$, which in turn means that $\beta$ is in $\varphi_{*}(G)$. In view of $\left(a_{5}\right)$, we obtain

$$
\varphi_{*}(G)=H_{k}^{\mathrm{alg}}\left(X_{0}, \mathbb{Z} / 2\right)
$$

The proof is complete.

Proof of Theorem 1.2. Let $T$ be the set of all integers $k$ such that $1 \leq k \leq m-1$ and the group $H_{m-k}(M, \mathbb{Z} / 2)$ is generated by $(m-k)$-dimensional smooth submanifolds of $M$ with trivial normal bundle.

Suppose $T$ is empty. If $m=0$, there is nothing to prove. If $m \geq 1$, then according to [26], there exists an irreducible algebraic model $X$ of the disjoint union of two copies of $M$. Let $X_{0}$ be the union of some connected components of $X$ such that $X_{0}$ is diffeomorphic to $M$. Then $\left(X, X_{0}\right)$ is a nice Nash algebraic model of $M$. Since $X$ is irreducible, we have $H_{k}^{\text {alg }}\left(X_{0}, \mathbb{Z} / 2\right)=0$ for $k \geq m$, as required.

Henceforth, we assume $T$ is nonempty, with

$$
T=\left\{k_{1}, \ldots, k_{s}\right\} .
$$

Each group $H^{k_{i}}(M, \mathbb{Z} / 2), 1 \leq i \leq s$, is generated by spherical cohomology classes. Hence, with notation as in Lemma 2.1, there exist a positive integer $d_{i}$ and a smooth map $f_{i}: M \rightarrow B^{k_{i}}(d)$ satisfying

$$
f_{i}(M) \subseteq B_{0}^{k_{i}}\left(d_{i}\right) \text { and } H^{k_{i}}(M, \mathbb{Z} / 2)=f_{i}^{*}\left(H^{k_{i}}\left(B^{k_{i}}\left(d_{i}\right), \mathbb{Z} / 2\right)\right) .
$$

The equality in $\left(b_{1}\right)$ implies that the induced homomorphism

$$
\left(f_{i}\right)_{*}: H_{k_{i}}(M, \mathbb{Z} / 2) \rightarrow H_{k_{i}}\left(B^{k_{i}}\left(d_{i}\right), \mathbb{Z} / 2\right) \text { is injective. }
$$

Moreover, we can find such a map $f_{i}$ with the additional property that it is constant on some open subset $U$ of $M$ which has a nonempty intersection with each connected component of $M$. Let $C$ be a 1-dimensional smooth submanifold of $M$ such that $C \subseteq U$, each connected component of $M$ contains a connected component of $C$, and the normal bundle of $C_{i}$ in $M$ is trivial. In particular, $f_{i} \mid C: C \rightarrow B^{k_{i}}\left(d_{i}\right)$ is a constant map. 
Set $\bar{M}:=(M \times\{0\}) \cup(M \times\{1\})$ and let $e: \bar{M} \rightarrow \mathbb{R}^{n}$ be a smooth embedding, $n \geq 2 m+1$, such that

$$
Y:=e((C \times\{0\}) \cup(C \times\{1\}))
$$

is a nonsingular irreducible algebraic subset of $\mathbb{R}^{n}$. Let $\bar{f}_{i}: e(\bar{M}) \rightarrow B^{k_{i}}\left(d_{i}\right)$ be defined by $\bar{f}_{i}(e(x, 0))=\bar{f}_{i}(e(x, 1))=f_{i}(x)$ for all $x$ in $M$. By construction, the map

$$
\bar{f}=\left(\bar{f}_{1}, \ldots, \bar{f}_{s}\right): e(\bar{M}) \rightarrow B:=B^{k_{1}}\left(d_{1}\right) \times \cdots \times B^{k_{s}}\left(d_{s}\right)
$$

represents 0 in the unoriented bordism group $\mathcal{N}_{*}(B)$. Moreover, the normal bundle of $Y$ in $e(\bar{M})$ is trivial, and $\bar{f} \mid Y: Y \rightarrow B$ is a constant map. By [3, Theorem 2.8.4], there exist a nonnegative integer $p$, a nonsingular algebraic subset $X$ of $\mathbb{R}^{n+p}$, a smooth diffeomorphism $\psi: e(\bar{M}) \rightarrow X$, and a regular map

$$
g=\left(g_{1}, \ldots, g_{s}\right): X \rightarrow B
$$

such that $Y \subseteq X, \psi(y)=y$ for all $y$ in $Y$, and $g$ is homotopic to $\bar{f} \circ \psi^{-1}$ (we regard $\mathbb{R}^{n}$ as a subset of $\mathbb{R}^{n+p}$, identifying $\mathbb{R}^{n}$ with $\left.\mathbb{R}^{n} \times\{0\} \subseteq \mathbb{R}^{n} \times \mathbb{R}^{p}=\mathbb{R}^{n+p}\right)$. Setting $X_{0}:=\psi(e(M \times\{0\}))$, we obtain a nice Nash algebraic model $\left(X, X_{0}\right)$ of $M$. Moreover, $X$ is irreducible since $Y$ is irreducible. In particular, $H_{k}^{\text {alg }}\left(X_{0}, \mathbb{Z} / 2\right)=0$ for $k \geq m$.

It remains to prove that $H_{k_{i}}^{\text {alg }}\left(X_{0}, \mathbb{Z} / 2\right)=0$ for $1 \leq i \leq s$. Define $\varphi: M \rightarrow X_{0}$ by $\varphi(x)=\psi(e(x, 0))$ for all $x$ in $M$. Then $\varphi$ is a smooth diffeomorphism, and $g_{i} \mid X_{0}$ is homotopic to $\left(f \circ \psi^{-1}\right) \mid X_{0}=f_{i} \circ \varphi^{-1}$. In particular, $g_{i}\left(X_{0}\right) \subseteq B_{0}^{k_{i}}\left(d_{i}\right)$ and, according to $\left(b_{2}\right)$, the induced homomorphism

$$
\left(g_{i} \mid X_{0}\right)_{*}: H_{k_{i}}\left(X_{0}, \mathbb{Z} / 2\right) \rightarrow H_{k_{i}}\left(B^{k_{i}}\left(d_{i}\right), \mathbb{Z} / 2\right)
$$

is injective. Hence, making use of Lemma 2.1, we get $H_{k_{i}}^{\text {alg }}\left(X_{0}, \mathbb{Z} / 2\right)=0$, as required.

\section{REFERENCES}

[1] S. Akbulut and H. King, The topology of real algebraic sets, Enseign. Math. 29(1983), 221261. MR719311 (86d:14016b)

[2] S. Akbulut and H. King, A relative Nash theorem, Trans. Amer. Math. Soc. 267(1981), 465-481. MR626484 (83b:58004)

[3] S. Akbulut and H. King, Topology of Real Algebraic Sets, Math. Sci. Res. Inst. Publ. 25, Springer, New York, 1992. MR1225577 (94m:57001)

[4] R. Benedetti and A. Tognoli, Approximation theorems in real algebraic geometry, Boll. Unione Mat. Ital. Suppl. (1980), no. 2, 209-228. MR675502 (84h:58004)

[5] R. Benedetti and A. Tognoli, On real algebraic vector bundles, Bull. Sci. Math. (2) 104(1980), 89-112. MR560747 (81e:14009)

[6] R. Benedetti and A. Tognoli, Remarks and counterexamples in the theory of real vector bundles and cycles, Géometrie algébrique réele et formes quadratiques. Lecture Notes in Math. 959, 198-211, Springer, Berlin, 1982. MR683134(85a:14018)

[7] J. Bochnak, M. Coste and M.-F. Roy, Real Algebraic Geometry, Ergeb. Math. Grenzgeb. (3) 36, Springer, Berlin, 1998. MR.1659509(2000a:14067)

[8] J. Bochnak and W. Kucharz, Algebraic models of smooth manifolds, Invent. Math. 97(1989), 585-611. MR.1005007 (91b:14076)

[9] J. Bochnak and W. Kucharz, Algebraic cycles and approximation theorems in real algebraic geometry, Trans. Amer. Math. Soc. 337(1993), 463-472. MR1091703 (93g:57033)

[10] J. Bochnak and W. Kucharz, On homology classes represented by real algebraic varieties, Banach Center Publications 44, 21-35, Warsaw, 1998. MR 1677394(2000b:14080)

[11] A. Borel and A. Haefliger, La classe d'homologie fondamentale d'un espace analytique, Bull. Soc. Math. France 89(1961), 461-513. MR0149503 (26:6990) 
[12] P. E. Conner, Differentiable Periodic Maps, 2nd ed., Lecture Notes in Math. 738, Springer, Berlin, 1979. MR548463 (81f:57018)

[13] J. Huisman and F. Mangolte, Every orientable Seifert 3-manifold is a real component of a uniruled algebraic variety, Topology 44(2005), 63-71. MR2104001 (2005h:14139)

[14] J. Huisman and F. Mangolte, Every connected sum of lens spaces is a real component of a uniruled algebraic variety, Ann. Inst. Fourier (Grenoble) 55(2005), 2475-2487. MR2207390 (2006k:57047)

[15] J. Kollár, The topology of real and complex algebraic varieties, Taniguchi Conference on Mathematics Nara '98, 127-145, Adv. Stud. Pure Math. 31, Math. Soc. Japan, Tokyo, 2001. MR.1865090 (2003a:14033)

[16] W. Kucharz, On homology classes of real algebraic sets, Invent. Math. 82(1985), 19-26. MR.808106 (87b:57025)

[17] W. Kucharz, Algebraic equivalence and homology classes of real algebraic cycles, Math. Nachr. 180(1996), 135-140. MR1397672 (97e:14009)

[18] W. Kucharz, Algebraic cycles and algebraic models of smooth manifolds, J. Algebraic Geometry 11(2002), 101-127. MR.1865915 (2002j:14065)

[19] W. Kucharz, Nash cohomology of smooth manifolds, Annal. Polonici Math. 87(2005), 193205. MR2208545 (2006m:14081)

[20] J. Nash, Real algebraic manifolds, Ann. of Math. (2) 56(1952), 405-421. MR0050928 $(14: 403 \mathrm{~b})$

[21] J.-J. Risler, Sur l'homologie des surfaces algébriques réelles. Géométrie algébrique réelle et formes quadratiques. Lecture Notes in Math. 959, 381-385, Springer, Berlin, 1982. MR683144 (84c:14019)

[22] R. Silhol, $A$ bound on the order of $H_{n-1}^{(a)}(X, \mathbb{Z} / 2)$ on a real algebraic variety. Géometrie algébrique réelle et formes quadratiques. Lecture Notes in Math. 959, 443-450, Springer, Berlin, 1982. MR683148 (84e:14024)

[23] E. Spanier, Algebraic Topology, McGraw-Hill, New York, 1966. MR0210112 (35:1007)

[24] R. Thom, Quelques propriétés globales des variétés différentiables, Comment. Math. Helv. 28(1954), 17-86. MR0061823 (15:890a)

[25] A. Tognoli, Su una congettura di Nash, Ann. Scuola Norm. Sup. Pisa Sci. Fis. Mat. 27(1973), 167-185. MR0396571(53:434)

[26] A. Tognoli, Une remarque sur les approximations en géométrie algébrique réelle. C. R. Acad. Sci. Paris Sér. I Math. 296(1983), 745-747. MR707333 (85b:14031)

Department of Mathematics and Statistics, University of New Mexico, Albuquerque, New MeXico 87131

E-mail address: kucharz@math.unm.edu 